\title{
Instability Tools and Reactive Motor Tasks: Effects of 8 Weeks Training Program on Motor Abilities in Healthy Women Over 65 Years Old
}

\author{
Sannicandro Italo ${ }^{1,2^{*}}$, Cofano Giacomo ${ }^{3}$, Rosa Anna Rosa ${ }^{2}$
}

${ }^{1}$ Clinical and Experimental Medicine Department, University of Foggia, Italy

${ }^{2}$ Master's Degree of Preventive and Adapted Physical Activity, University of Foggia, Italy

${ }^{3}$ Master's Degree of Motor and Sport Science, University of Foggia, Italy

DOI: $10.36348 /$ jaspe.2020.v03i06.002 | Received: 31.05 .2020 | Accepted: 08.06.2020 | Published: 30.06 .2020

*Corresponding author: Sannicandro Italo

\section{Abstract}

Background: The balance ability represents a significant senior health factor, in particular for the individual maintenance autonomy and individual mobility. In the literature an open problem is understand the walking exercises and one-foot stance exercises effectiveness performed using tools or enviroments/conditions that give an unpredictable instability. The aim of this study is to verify the effects of motor task through instability tools and through reactive motor tasks on motor abilities in older women. Methods: A sample of 58 elderly women $(67.9 \pm 5.6$ years, $59.6 \pm 7.4 \mathrm{~kg}, 156.1 \pm 5.2)$ participated in the investigation and divided randomly into 2 groups: $n=27$ were included in the instability training group (IG) while $\mathrm{n}=26$ were included in the control group (CG). The instability training period lasted 8 weeks ( 3 sessions/week, total= 24 sessions of about $50 \mathrm{~min})$. Results: The most significant interactions between program type and time in the 8-foot-upand-go $(\mathrm{p}=<.05), 2$ Minutes Step test $(\mathrm{p}<.01)$, Arm Curl test $(\mathrm{p}<.005)$, Chair stand test $(\mathrm{p}<.01)$, Single Leg Stance test right foot $(\mathrm{p}<.05)$ and left $(\mathrm{p}<.005), 3-\mathrm{m}$ Backwards Walk test $(\mathrm{p}<.005)$, Tandem Walk test $(\mathrm{p}<.005)$. Conclusions: The tools and the tasks, that generate instability and the structuring of an environment that determines sudden disturbances, increase the senior over 65 years old balance ability.

Keywords: Balance, perturbation training, motor abilities, older adult, unstability, reactive.

Copyright @ 2020: This is an open-access article distributed under the terms of the Creative Commons Attribution license which permits unrestricted use, distribution, and reproduction in any medium for non-commercial use (NonCommercial, or CC-BY-NC) provided the original author and source are credited.

\section{INTRODUCTION}

The industrialized societies are subject to witnessing an increase of senior citizen population. This increase in over 65 senior's leads to many health age dependent problems and health care system sustainability [1].

Therefore, the balance ability represents a significant senior health factor, in particular for the individual maintenance autonomy and individual mobility [2-4].

Furthermore, in over 65 years old, balance and walking influence each other and the difficulties become more pronounced during the simultaneous execution of motor and cognitive tasks [4-7].

Infact, it is mentioning that the balance control is motor task-dependent [8-12]. Therefore, the design of the motor tasks to increase the capacity for balance must consider in which environment the senior will be able to use these learning and through which strategies.
After a postural perturbation, infact, the balance recovery can be realized by ankle or/and hip strategy, compensatory step, grabbing a support and counter rotations of body segments $[13,14]$.

The no-specific physical activity seems to be suitable to increase the balance [15-18], but a more specific methodology, called in literature perturbationbased balance training, seems more effective [19].

The perturbation-based balance training has so far mainly used some types of perturbations: therapist perturbation, cable perturbation on traditional treadmill, specific treadmill with belt acceleration perturbation computer assisted, moveable platform or perturbations caused by moveable platform and therapist [19].

However, today the rapid diffusion of tools, that generate instability and perturbations (rubber bands, sandbags, waterbags, i.e.) during the movement 
execution, makes it possible to structure this type of motor tasks with greater simplicity in the gym.

The availability of this new type of tools and specific tasks allows to structure new exercises much more similar to the senior daily life movement.

These movements infact are carried out simultaneously on the three movement planes, unlike what often happened in the balance training programs in which the sagittal plane tasks have been boosted [20].

However, in the literature an open problem is to understand the effectiveness of walking exercises combined with monopodalic support exercises performed with the use of tools or variable environmental conditions that generate sudden instability.

The structuring of motor tasks of contextual/situational type also allows to create exercises very close to what occurs in reality, where perturbations and variations of movement are not proactive but reactive [2, 19-21].

The reactive control mechanism that is different from the proactive control balance mechanism primarily relies on the somatosensory and vestibular systems to determine the extent and type of the stimulus (threat) and to trigger appropriately scaled postural responses [2,19] These postural responses are mainly polysynaptic spinal reflexes and supraspinal responses [20, 22-24].

The aim of this study is verify the effects of motor tasks through instability tools and through reactive motor tasks on motor abilities in older women versus no-specific physical activities and no-specific exercises.

\section{EXPERIMENTAL SECTION Sample}

A sample of 53 (originally, $n=58$ ) women older adults $(67.9 \pm 5.6$ years, $59.6 \pm 7.4 \mathrm{~kg}, 156.1 \pm 5.2)$ participated in the investigation; the sample was divided by randomizing within blocks into 2 groups: $n=27$ were included in the instability training group (IG) while $n=$ 26 were included in the control group (CG); $n=5$ were excluded from the study because they did not complete the assessments (for personal and family reasons). The subjects included in the study did not exhibit cardiovascular, metabolic or skeletal muscle pathologies, nor did they undergo pharmacological treatment. All subjects have been informed in advance about the proposed training and potential injury risk; the study was conducted acknowledging the principles presented in the Helsinki Declaration.

\section{Materials}

The following functional tests were used to evaluate motor capacity:

- Chair stand test was presented to evaluate the lower limbs strength; the subject is invited to get up and sit as many times in the 30 -second interval. The detector records the number of repetitions performed [25];

- 8-foot-up-and-go test was presented to evaluate dynamic balance in a daily task; the assessment involves getting out of a chair, walking 2.44 meters to and around a cone, and returning to the chair in the shortest time possible. The time needed to perform the task measured with $1 / 10$-second accuracy constitutes the test's result [25];

- Single Leg Stance test was presented for static balance evaluation; the subject standing on one foot, with his hands on the chest and with open eyes, is invited to maintain the position assigned taking care not to tilt the bust, abduct the lower limb in suspension or touching the ground. It detects the time at which the subject is able to keep the assigned task. The test was performed on the favorite limb, and measured with $1 / 10$ second accuracy [26];

- 2 Minutes Step test was used to evaluate aerobic ability; the subject performs the greater number of thigh flexions on the pelvis without moving. It detects the number of movements performed in 2 minutes [25].

- Arm Curl test (preferred upper limb), was used to evaluate upper body strength; the subject performs the greater number of curl movement using a dumbell of $2.3 \mathrm{~kg}$ in the 30 -second interval. It detects the number of movements performed in 30 second-interval [25].

- 3-m Backwards Walk test was used to evaluate dynamic balance during walking backwards; the subject, walks backwards for 3 meters and the task were measured with $1 / 10$ second accuracy. The partecipant performs two trials and the average time was calculated [27].

- Tandem walk test was used to evaluate dynamic balance during a specific walking task performed on a line ( $2 \mathrm{~m}$ length, $5 \mathrm{~cm}$ wide): the subject was instructed to place one foot behind the other, each time making sure that the toe of swing foot was placed directly behind the heel of stance foot. The subject was walk as fast and comfortable as possible without falling down [28,29].

\section{Protocol}

The training period lasted 8 weeks, 3 sessions a week for a total of 24 sessions of about 50 minutes each in accordance with the literature [30].

Before and after the training period (T0 and T1), there were established two evaluation sessions on motor skills and anthropometric variables that provided Chair stand test, Arm Curl test, Single leg stance test, 8- 
foot-up-and-go test, 3-m Backwards Walk and 2 Minutes Step test. These assessment sessions were conducted by expert preventive and adapted physical activity graduates.

The test order was random, with the exception of the 2 minute step test, which is always the last test, as well as the indications provided by protocol [25].

Each training session provided an initial 10 minute warm up phase for both groups, while the central phases were differentiated according to the assigned plans (Table 1); the subjects motor level skills and the literature indications, with particular reference to senior people, determined the choice of the intensity assigned to $(3 / \mathrm{w}, 50 \mathrm{~min} / \mathrm{session})$ of static balance training and walking tasks using instability tools (Slashpipe, Slashpipe GmbH, Essen, Germany) and reactive motor tasks. The exercise order presentation was randomized in each session.The control group followed recreational activities and no-specific physical activity (free walking, mobility, flexibility and gym with music), without the precise determination of the motor load (or "free load") for the same time length.

Table-1: The different training assigned for IG and CG.

\begin{tabular}{|c|c|}
\hline Central phase Instability training Group (IG) & $\begin{array}{l}\text { Central phase Control } \\
\text { Group (CG) }\end{array}$ \\
\hline 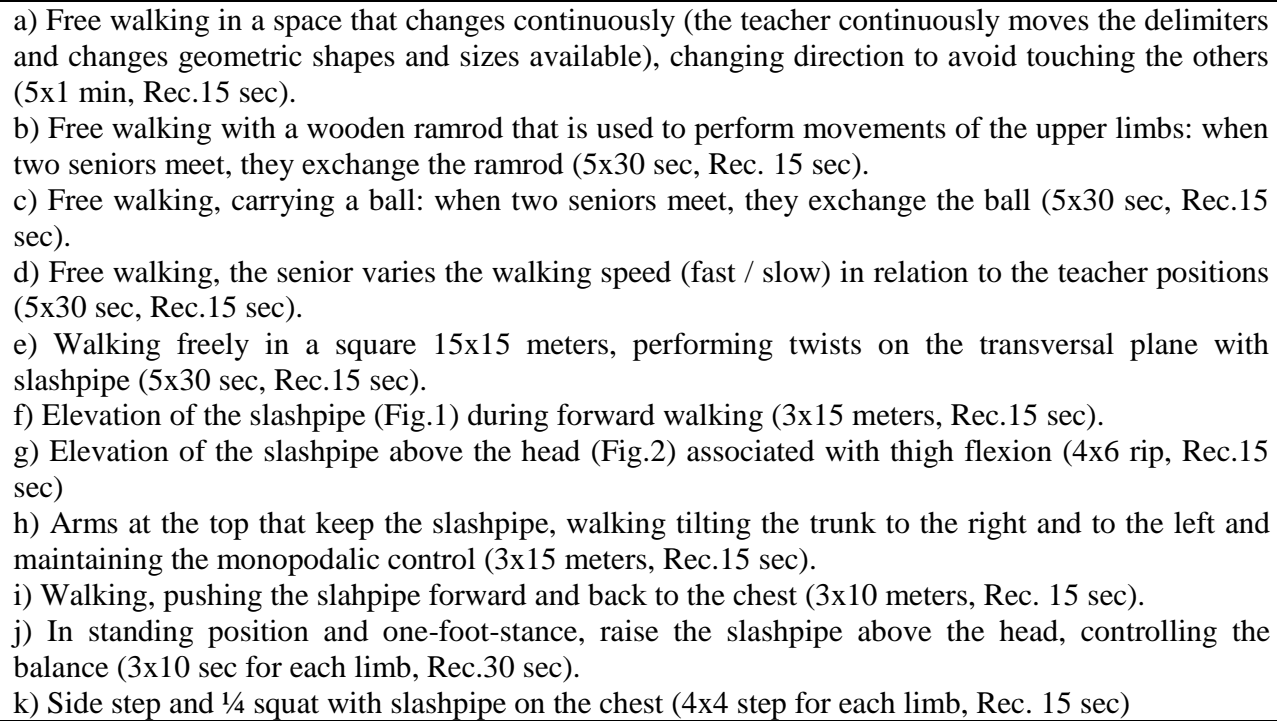 & $\begin{array}{ll}\text { a) } & \text { Free talk walking } \\
\text { b) Joint mobility } \\
\text { c) Flexibility } \\
\text { d) } \text { Gym with music } \\
\text { (recreational free } \\
\text { dance) }\end{array}$ \\
\hline
\end{tabular}

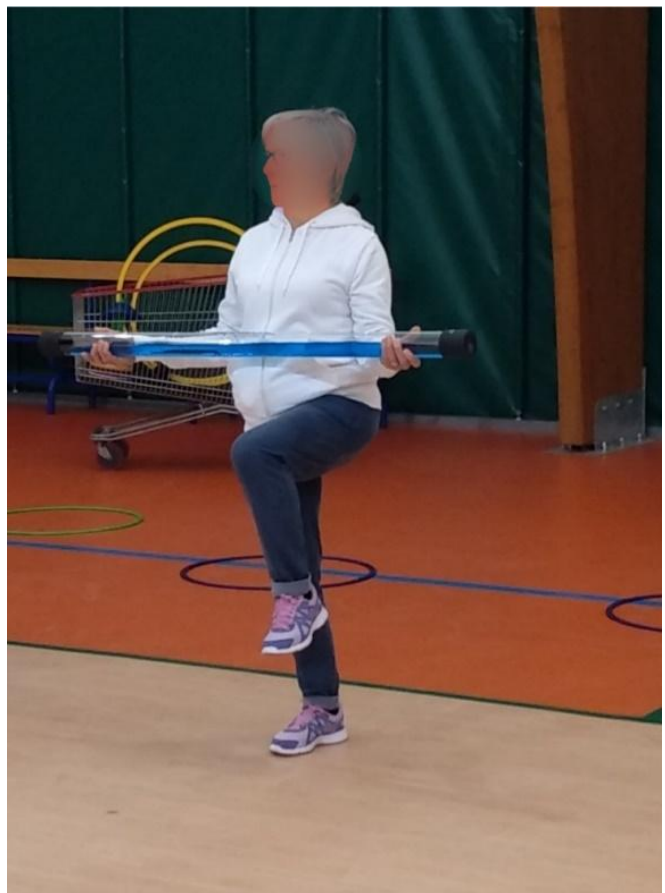

Fig-1

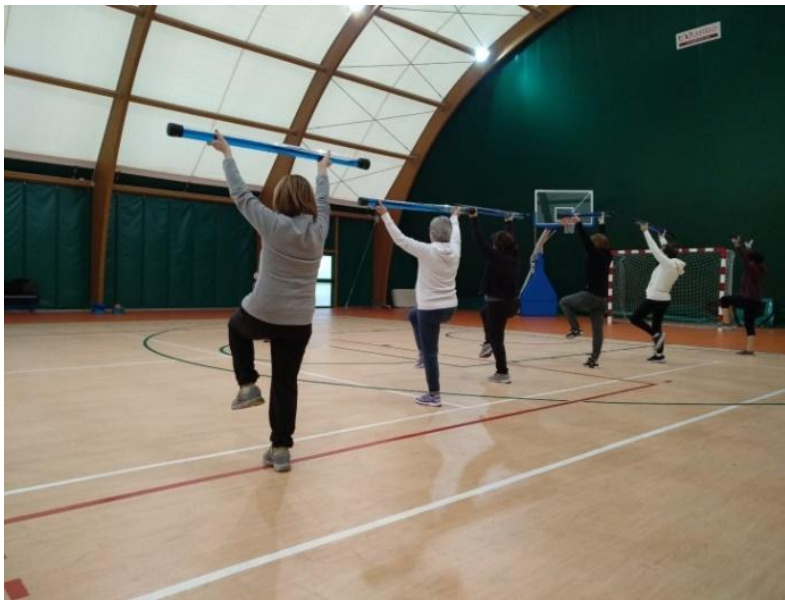

Fig-2

\section{STATISTICAL ANALYSIS}

For all the values obtained, the descriptive statistic (mean, standard deviation) was determined. A 2 (time) $\mathrm{x} 2$ (group) mixed-model ANOVA was used to examine the data of both groups together (IG and CG) in the pre- and post-training time points (T0 vs T1) in order to determine the main and interactive effects of training. Statistical significance was set at $\mathrm{p}<0.05$. 


\section{RESULTS}

The results revealed a change in test scores across the two time periods (pre-intervention, post intervention) in the following tests:

- 8 -foot-up-and-go test $F(1,50)=10.73, \mathrm{p}<.005$, partial $\eta^{2} .17$, effect size large [31,32];

- 2 Minutes Step test $F(1,50)=44.74, \mathrm{p}<.005$, partial $\eta^{2} .47$, effect size large [31];

- $\quad$ Arm Curl test $\mathrm{F}(1,50)=42.37, \mathrm{p}<.005$, partial $\eta^{2}$ .45 , effect size large [31];

- $\quad$ Chair stand test $F(1,50)=28.57, \mathrm{p}<.005$, partial $\eta^{2}$ .36 , effect size large [31];

- $\quad$ Single Leg Stance test right $F(1,50)=11.93$, $\mathrm{p}<.005$, partial $\eta^{2} .19$, effect size large [31];

- $\quad$ Tandem Walk $F(1,50)=2.84, p<.005$, partial $\eta^{2} .19$ , effect size large [31].

The main effect in test scores comparing the two groups (IG: instability group, CG: no-specific physical activities) was significant in the following tests:

- 8-foot-up-and-go test $\mathrm{F}(1,50)=41.68, \mathrm{p}<.005$, partial $\eta^{2} .45$

- 2 Minutes Step test $F(1,50)=28.68, \mathrm{p}<.005$, partial $\eta^{2} .36$

- Arm Curl test $\mathrm{F}(1,50)=75.17, \mathrm{p}<.005$, partial $\eta^{2}$ .60
- Chair stand test $\mathrm{F}(1,50)=54.63, \mathrm{p}<.005$, partial $\eta^{2}$ .52

- $\quad$ Single Leg Stance test right $F(1,50)=8.87, \mathrm{p}<.005$, partial $\eta^{2} .15$

- $\quad$ Single Leg Stance test left $F(1,50)=4.84, p<.05$, partial $\eta^{2} .08$

- 3-m Backwards Walk test $F(1,50)=93.04, p<.005$, partial $\eta^{2} .65$

- $\quad$ Tandem Walk test $F(1,50)=63.25, \mathrm{p}<.005$, partial $\eta^{2} .57$.

The most significant interactions between program type and time in the following tests:

- 8-foot-up-and-go test $\mathrm{F}(1,50)=5.61, \mathrm{p}=<.05$, partial $\eta^{2} .10$

- 2 Minutes Step test $F(1,50)=6.31, \mathrm{p}<.01$, partial $\eta^{2}$ .11

- Arm Curl test $F(1,50)=9.32, p<.005$, partial $\eta^{2} .11$

- Chair stand test $F(1,50)=8.14, p<.01$, partial $\eta^{2} .14$

- Single Leg Stance test right $F(1,50)=5.21, \mathrm{p}<.05$, partial $\eta^{2} .09$

- $\quad$ Single Leg Stance test left $F(1,50)=10.39, \mathrm{p}<.005$, partial $\eta^{2} .17$

- 3-m Backwards Walk test $F(1,50)=67.95, p<.005$, partial $\eta^{2} .57$

- $\quad$ Tandem Walk test $F(1,50)=15.04, \mathrm{p}<.005$, partial $\eta^{2} .24$.

The results are summarized in Table 2 and in Table 3.

Table-2: Means, standard deviation, percentage performance increases of Physical Fitness Tests

\begin{tabular}{|c|c|c|c|c|}
\hline & & T0 & T1 & \\
\hline Variable & groupe & Mean \pm SD & Mean \pm SD & $\%$ increases \\
\hline \multirow[t]{2}{*}{ 8-foot-up-and-go test (sec) } & $\mathrm{IG}$ & $6.6 \pm 1.9$ & $4.8 \pm 0.7$ & -27.2 \\
\hline & CG & $6.1 \pm 0.5$ & $6.6 \pm 0.7$ & +7.6 \\
\hline \multirow[t]{2}{*}{2 Minutes Step test (reps) } & IG & $121.2 \pm 40.7$ & $178.6 \pm 42.7$ & +32.2 \\
\hline & CG & $128.5 \pm 15.4$ & $134.9 \pm 7.1$ & +4.8 \\
\hline \multirow[t]{2}{*}{ Arm Curl test (reps) } & IG & $17.4 \pm 3.7$ & $21.7 \pm 4.7$ & +19.2 \\
\hline & CG & $17.4 \pm 3.6$ & $16.6 \pm 3.9$ & -4.6 \\
\hline \multirow[t]{2}{*}{ Chair stand test (reps) } & IG & $15.6 \pm 3.01$ & $18.6 \pm 3.6$ & +16.2 \\
\hline & CG & $15.1 \pm 3.8$ & $14.5 \pm 2.5$ & -3.9 \\
\hline \multirow{2}{*}{$\begin{array}{l}\text { Single Leg Stance test right } \\
(\mathrm{sec})\end{array}$} & IG & $19.2 \pm 7.9$ & $26.10 \pm 5.1$ & +26.5 \\
\hline & CG & $19.1 \pm 7.9$ & $18.7 \pm 6.4$ & -2.1 \\
\hline \multirow{2}{*}{$\begin{array}{c}\text { Single Leg Stance test } \\
\text { left }(\mathrm{sec})\end{array}$} & IG & $20.7 \pm 7.9$ & $24.7 \pm 4.9$ & +16.2 \\
\hline & CG & $19.5 \pm 3.8$ & $18.1 \pm 4.8$ & -7.2 \\
\hline \multirow[t]{2}{*}{ 3-m Backwards Walk test (sec) } & IG & $4.4 \pm 0.8$ & $2.8 \pm 0.6$ & -26.4 \\
\hline & CG & $4.8 \pm 0.7$ & $5.9 \pm 1.5$ & +18.7 \\
\hline \multirow[t]{2}{*}{ Tandem Walk test (sec) } & IG & $9.5 \pm 2.6$ & $6.4 \pm 1.7$ & -22.7 \\
\hline & CG & $9.5 \pm 2.5$ & $10.7 \pm 1.5$ & +11.2 \\
\hline
\end{tabular}


Table-3: Analyses of variance

\begin{tabular}{|c|c|c|c|c|c|}
\hline Variables & Source & $F(1,50)$ & $p$ & Partial $\eta^{2}$ & Cohen's $f$ \\
\hline \multirow[t]{3}{*}{ 8-foot-up-and-go test (sec) } & Time & $10.73 * * * *$ & .002 & .17 & 0.43 \\
\hline & Group & $41.68 * * * *$ & .000 & .45 & \\
\hline & Time x Group & $5.61 *$ & .02 & .10 & \\
\hline \multirow[t]{3}{*}{2 Minutes Step test (reps) } & Time & $44.74 * * * *$ & .000 & .47 & 0.91 \\
\hline & Group & $28.68 * * * *$ & .000 & .36 & \\
\hline & Time x Group & $6.31 *$ & .01 & .11 & \\
\hline \multirow[t]{3}{*}{ Arm Curl test (reps) } & Time & $42.37 * * * *$ & .000 & .45 & 0.89 \\
\hline & Group & $75.17 * * * *$ & .000 & .60 & \\
\hline & Time x Group & $9.32 * * * *$ & .004 & .15 & \\
\hline \multirow[t]{3}{*}{ Chair stand test (reps) } & Time & $28.57 * * * *$ & .000 & .36 & 0.72 \\
\hline & Group & $54.63 * * * *$ & .000 & .52 & \\
\hline & Time x Group & $8.14 * *$ & .006 & .14 & \\
\hline \multirow[t]{3}{*}{ Single Leg Stance test right $(\mathrm{sec})$} & Time & $11.93 * * * *$ & .001 & .19 & 0.45 \\
\hline & Group & $8.87 * * * *$ & .004 & .15 & \\
\hline & Time x Group & $5.21 *$ & .02 & .09 & \\
\hline \multirow[t]{3}{*}{ Single Leg Stance test left $(\mathrm{sec})$} & Time & 2.35 & .13 & .04 & 0.44 \\
\hline & Group & $4.84 *$ & .03 & .08 & \\
\hline & Time x Group & $10.39 * * * *$ & .002 & .17 & \\
\hline \multirow[t]{3}{*}{ 3-m Backwards Walk test (sec) } & Time & 2.84 & .98 & .05 & 0.68 \\
\hline & Group & $93.04 * * * *$ & .000 & .65 & \\
\hline & Time x Group & $67.95 * * * *$ & .000 & .57 & \\
\hline \multirow[t]{3}{*}{ Tandem Walk test $(\mathrm{sec})$} & Time & $12.27 * * * *$ & .001 & .20 & 0.46 \\
\hline & Group & $63.25 * * * *$ & .000 & .57 & \\
\hline & Time x Group & $15.04 * * * *$ & .000 & .24 & \\
\hline
\end{tabular}

\section{DISCUSSION}

The study aimed to describe and understand the effects of introducing instability tools and reactive motor tasks on over 65 aged motor capabilities. In everyday life the environment in which the senior moves is extremely variable; in particular way, this variability requires a continuous variation in the movement execution and walking [7].

Some authors have shown the sensory-motor integration through visual training may be an important component for dynamic balance improvements in older adults during functional integrative gait tasks $[2,33]$.

The lower limb strength evaluation in the IG showed a statistically significant increase in performance (approximately $+16 \%, p=0.006$, ES large) at the end of the 8 weeks: the getting up from the chair skill in fact is strongly influenced by postural control and balance [34,35]. The performance gains obtained in the IG in the Arm Curl test (approximately $+19 \%, p=0.004$, ES large) can be explained in consideration of the choice of some motor tasks that involved the use of the Slashpipe of about $5 \mathrm{~kg}$ : this external resistance, even if modest, it may have represented an effective load to increase the upper limb strength in the elderly over 65 years old. The assessment of walking speed through 8 -foot-up-and-go in the pre-posttest comparison in IG showed a significant increase (approximately $+27 \%, p=0.000$, ES large): this increase is consistent with what was identified in literature following balance training programs [30].
It suggests that speed walking increased, not only because older adults proactively improved their movement stability, but also because they improve their reactive response so the loss of balance resulting in walking motor task decreases, as indicated in the literature for many years $[2,36]$.

The statistically significant performance increases achieved in the 3-m Backwards Walk test (approximately 26\%, p $=0.000$, ES large) suggests that the proposed program allows transferring even in skills (walking backwards) not specifically trained during the 8 weeks.

The free walking tasks in which the space available for the exercise was constantly varied and those which included the exchange of tools have probably suddenly requested to walk backwards and / or, in any case, in different directions.

The performance increases obtained in the Single-leg stance test for both limbs (left $+16 \%, \mathrm{p}=$ 0.002 ES large, right $+26 \%, p=0.004$, ES large) are lower than those described in the literature $[30,37]$.

Most likely the prevalent use of dynamic balance motor tasks may have limited the static balance performance assessment; all this confirms that balance control is motor-task-dependent $[9,10,11]$.

The statistically significant performance increases achieved in the Tandem Walk test (approximately $+22 \%, \mathrm{p}=0.000 \mathrm{ES}$ large), which is a 
dynamic equilibrium test, seems to confirm this hypothesis since the advantages obtained are superior to those already seen in literature: in fact, so far, have been observed performance increases of approximately $1.3 \%$ and $14.6 \%[30,36,38,39]$.

The study allowed to understand the advantages obtainable from the use of motor tasks that call for reactive control, through the sudden perturbations of the tools used in this study or through the creation of executive constraints in the environment that require rapid and unexpected changes in the execution of the programmed movement.

\section{CONCLUSIONS}

The training programs structuring that introduce the older womens into a variable environment or that requires instability control allows recreating similar and specific reality conditions [10-12]. In which they must move. In fact, the reactive control to sudden perturbations is the most functional to the environment demands in which the elderlies live and interact [7]. However, the proposed exercises can be further expanded (in numerical and difficulty terms) to be able to better respond to the methodological rationale indicated in the literature [40].

However, this study has some limitations: there was no minimum adherence required to allow subjects to be included/excluded from the study; and there were no differences between those who showed greater or lesser compliance.

\section{Authors' contribution}

Italo Sannicandro contributed to research conception and design, data acquisition, data analysis and interpretation, writing and critical review of the manuscript. Giacomo Cofano contributed to data acquisition and interpretation. Rosa Anna Rosa contributed to research design and statistical analysis.

\section{Founding}

This research was authorized and supported by the Department of Clinical and Experimental Medicine of the University of Foggia (Italy) through research grants aimed to program implementing. This research received no specific grant from any commercial, or profit sectors.

\section{Competing Interests} interests exist.

The authors have declared that no competing

\section{REFERENCES}

1. Reinhardt, U. E. (2003). Does the aging of the population really drive the demand for health care?. Health Affairs, 22(6), 27-39.

2. Paquette, M. R., Li, Y., Hoekstra, J., \& Bravo, J. (2015). An 8-week reactive balance training program in older healthy adults: A preliminary investigation. Journal of Sport and Health Science, 4(3), 263-269.

3. Gomez, F., Curcio, C. L., Suriyaarachchi, P., Demontiero, O., \& Duque, G. (2013). Differing approaches to falls and fracture prevention between Australia and Colombia. Clinical interventions in aging, 8, 61 .

4. Granacher, U., Bridenbaugh, S. A., Muehlbauer, T., Wehrle, A., \& Kressig, R. W. (2011). Agerelated effects on postural control under multi-task conditions. Gerontology, 57(3), 247-255.

5. Granacher, U., Muehlbauer, T., \& Gruber, M. (2012). A qualitative review of balance and strength performance in healthy older adults: impact for testing and training. Journal of aging research, 2012.

6. Granacher, U., Gruber, M., \& Gollhofer, A. (2010). Force production capacity and functional reflex activity in young and elderly men. Aging clinical and experimental research, 22(5-6), 374-382.

7. Dunsky, A. (2019). The effect of balance and coordination exercises on quality of life in older adults: a mini-review. Frontiers in aging neuroscience, 11 .

8. Muehlbauer, T., Besemer, C., Wehrle, A., Gollhofer, A., \& Granacher, U. (2012). Relationship between strength, power and balance performance in seniors. Gerontology, 58(6), 504512.

9. Grabiner, M. D., Crenshaw, J. R., Hurt, C. P., Rosenblatt, N. J., \& Troy, K. L. (2014). Exercisebased fall prevention: can you be a bit more specific?. Exercise and sport sciences reviews, 42(4), 161-168.

10. Giboin, L. S., Gruber, M., \& Kramer, A. (2015). Task-specificity of balance training. Human movement science, 44, 22-31.

11. Kümmel, J., Kramer, A., Giboin, L. S., \& Gruber, M. (2016). Specificity of balance training in healthy individuals: a systematic review and metaanalysis. Sports Medicine, 46(9), 1261-1271.

12. Ringhof, S., \& Stein, T. (2018). Biomechanical assessment of dynamic balance: Specificity of different balance tests. Human movement science, 58, 140-147.

13. Maki, B. E., \& McIlroy, W. E. (2005). Change-insupport balance reactions in older persons: an emerging research area of clinical importance. Neurologic Clinics, 23(3), 751-783.

14. Maki, B. E., \& McIlroy, W. E. (2005). Change-insupport balance reactions in older persons: an emerging research area of clinical importance. Neurologic Clinics, 23(3), 751-783.

15. Oddsson, L. I., Boissy, P., \& Melzer, I. (2007). How to improve gait and balance function in elderly individuals - compliance with principles of training. European Review of Aging and physical activity, 4(1), 15.

16. Mansfield, A., Peters, A. L., Liu, B. A., \& Maki, B. E. (2010). Effect of a perturbation-based balance 
training program on compensatory stepping and grasping reactions in older adults: a randomized controlled trial. Physical therapy, 90(4), 476-491.

17. Carty, C. P., Cronin, N. J., Nicholson, D., Lichtwark, G. A., Mills, P. M., Kerr, G., ... \& Barrett, R. S. (2015). Reactive stepping behaviour in response to forward loss of balance predicts future falls in community-dwelling older adults. Age and ageing, 44(1), 109-115.

18. Sannicandro, I., Colella, D., Rosa, A.R., Manno, R.(2008). Motor load modulation in adults and elderly people: effects of different training protocols on strength, flexibility and endurance, Medicina dello Sport, 61:443-454.

19. Gerards, M. H., McCrum, C., Mansfield, A., \& Meijer, K. (2017). Perturbation- based balance training for falls reduction among older adults: Current evidence and implications for clinical practice. Geriatrics \& gerontology international, 17(12), 2294-2303.

20. Woollacott, M. H., \& Pei-Fang, T. (1997). Balance control during walking in the older adult: research and its implications. Physical therapy, 77(6), 646.

21. Shimada, H., Obuchi, S., Furuna, T., \& Suzuki, T. (2004). New intervention program for preventing falls among frail elderly people: the effects of perturbed walking exercise using a bilateral separated treadmill. American journal of physical medicine \& rehabilitation, 83(7), 493-499.

22. Patla, A. E. (1993). Age-related changes in visually guided locomotion over different terrains: major issues. In Sensorimotor impairment in the elderly (pp. 231-252). Springer, Dordrecht.

23. Dietz, V., Quintern, J., \& Berger, W. (1984). Corrective reactions to stumbling in man: functional significance of spinal and transcortical reflexes. Neuroscience letters, 44(2), 131-135.

24. Nashner, L. M. (1980). Balance adjustments of humans perturbed while walking. Journal of Neurophysiology, 44(4), 650-664.

25. Rikli, R. E., \& Jones, C. J. (1999). Functional fitness normative scores for community-residing older adults, ages 60-94. Journal of aging and physical activity, 7, 162-181.

26. Bohannon, R. W. (2006). Single limb stance times: a descriptive meta-analysis of data from individuals at least 60 years of age. Topics in Geriatric Rehabilitation, 22(1), 70-77.

27. Carter, V., Jain, T., James, J., Cornwall, M., Aldrich, A., \& de Heer, H. D. (2019). The 3-m backwards walk and retrospective falls: diagnostic accuracy of a novel clinical measure. Journal of geriatric physical therapy, 42(4), 249-255.

28. Robertson, M., \& Gregory, R. (2017). Concurrent validation of the tandem walk test as a measure of dynamic walking balance in a healthy population.

29. Cohen, H. S., Stitz, J., Sangi- Haghpeykar, H., Williams, S. P., Mulavara, A. P., Peters, B. T., \&
Bloomberg, J. J. (2018). Tandem walking as a quick screening test for vestibular disorders. The Laryngoscope, 128(7), 1687-1691.

30. Lesinski, M., Hortobágyi, T., Muehlbauer, T., Gollhofer, A., \& Granacher, U. (2015). Effects of balance training on balance performance in healthy older adults: a systematic review and metaanalysis. Sports medicine, 45(12), 1721-1738.

31. Cohen, J. (2013). Statistical power analysis for the behavioral sciences. Academic press.

32. Lakens, D. (2013). Calculating and reporting effect sizes to facilitate cumulative science: a practical primer for t-tests and ANOVAs. Frontiers in psychology, 4, 863.

33. Reed-Jones, R. J., Dorgo, S., Hitchings, M. K., \& Bader, J. O. (2012). Vision and agility training in community dwelling older adults: Incorporating visual training into programs for fall prevention. Gait \& posture, 35(4), 585-589.

34. Vaidya, T., Chambellan, A., \& de Bisschop, C. (2017). Sit-to-stand tests for COPD: A literature review. Respiratory medicine, 128, 70-77.

35. Pozaic, T., Lindemann, U., Grebe, A. K., \& Stork, W. (2016). Sit-to-stand transition reveals acute fall risk in activities of daily living. IEEE journal of translational engineering in health and medicine, 4, 1-11.

36. Piao, Y. J., Kim, K., Yu, M., Kwon, T. K., Kim, D. W., \& Kim, N. G. (2009). Analysis on training effects of postural control for elderly adults. International Journal of Precision Engineering and Manufacturing, 10(3), 133-139.

37. Hafström, A., Malmström, E. M., Terdèn, J., Fransson, P. A., \& Magnusson, M. (2016). Improved balance confidence and stability for elderly after 6 weeks of a multimodal selfadministered balance-enhancing exercise program: A randomized single arm crossover study. Gerontology and geriatric medicine, 2 , 2333721416644149.

38. Pfeifer, K., Ruhleder, M., Brettmann, K., \& Banzer, W. (2001). Effekte eines koordinationsbetonten Bewegungsprogramms zur Aufrechterhaltung der Alltagsmotorik im Alter. Deutsche Zeitschrift für Sportmedizin, 52(4), 129-135.

39. Maughan, K. K., Lowry, K. A., Franke, W. D., \& Smiley-Oyen, A. L. (2012). The dose-response relationship of balance training in physically active older adults. Journal of aging and physical activity, 20(4), 442-455.

40. Halvarsson, A., Dohrn, I. M., \& Ståhle, A. (2015). Taking balance training for older adults one step further: the rationale for and a description of a proven balance training programme. Clinical rehabilitation, 29(5), 417-425. 